\title{
Smart city smartness measurement based on KPI for developing countries
}

\author{
Pape Abdoulaye BARRO ${ }^{1,2}[0000-0001-9715-9234]$ \\ ${ }^{1}$ Intitute of Mathematics and Phisical Sciences (IMSP), Porto-Novo 3730-301, Benin \\ 2 T/ICT4D Laboratory of the Abdus Salam International Centre for Theoretical \\ Physics (ICTP), 34151 Trieste, Italy \\ pape.barro@imsp-uac.org \\ pape.abdoulaye.barro@gmail.com
}

\begin{abstract}
A smart city is a futuristic approach that evolves with new technologies. It can start as a simple community and evolve into future cities. In most developing countries, where the population growth is faster, and where the organization and the urbanization are slower, there is a serious need for a framework for progressive change towards sustainable cities. This article discusses the level of intelligence of smart city components and proposes indicators as well as a methodology for measuring intelligence based on the objectives to be achieved as well as a certain number of technical requirements and some cognitive processes implemented.
\end{abstract}

Keywords: Internet of Things for development (IoT4D); sustainability; smart \& futures cities; smart city components (SMART-X); Key Performance Indicators (KPI); cognitive processes

\section{Introduction}

There is abundant literature on the concept of smartness of a city that mainly refers to the intelligence of at least one of its components. The smartness commonly refers to the mental and cognitive abilities of an individual or others to solve a problem or to adapt in a given environment. However, the concept can be more complex and implies an integrated intelligence in an object (a component of a city), thus needs particular analysis. Consequently, it is difficult to find a common definition of the smart city paradigm in the literature [1].

Indeed, smartness can be measured and depends on the means used for its assessment. In the literature, these means are commonly called indicators [2], [3], [4], [5], and [6]. According to the ITU (International Telecommunication Union) [2], the indicators will enable cities to measure their progress over time, compare their performance to other cities and in meeting the Sustainable Development Goals (SDGs) at the city level. In the case of a city, we will refer to the smart component (or smart sub-component), when the indicators return values or acceptable data. A component or sub-component can be linked to sustainability without bearing the intelligence part. However, the intelligence comes into play 
when it involves Information and Communications Technologies (ICT) and their impact on the system. It is admitted as in [6], that technology was, and is still at the core of the Barcelona urban-development model and essential crosswise tool supporting the innovation process. Furthermore, [6] states that Barcelona's key smart projects reflected and continue to reflect the strategic use of technology in development of a smart city, and, today more than ever, cities require strategic planning. It is the only way they can embrace pathways to innovation and prioritize what is most important for their future [4], [5]. Moreover, it is admitted that each city is unique and unrepeatable and has its own needs and opportunities, so it must design its own plan, set its priorities and be flexible enough to adapt to changes.

According to the components of the smart city that range from smart urbanization to smart housing, defined in the "ideological framework" and taking into account the "technological framework" proposed as an approach for Africa[14], [15], it becomes possible to measure the degree of "smartness" of each component, taking into account the foreseen objectives and their actual impact. The overall objective is to come up with a simplified set of indicators that can, as much as possible, take into account the developing countries requirements and needs, with a particular focus on Africa. The developed countries situation is different in the sense that in most cases, the underlying infrastructure, on which they will build their evolution, is already in place. In the latter case, indicators focus more on objectivity. Of course, for an object or component to be intelligent, it must implement, in addition to its technology, certain cognitive processes such as knowledge, learning, reasoning, prediction, automatic correction, etc., and this, for better decision-making.

This document is divided into seven sections. Sections 2, 3 and 4 present respectively the context of the study, the research methodology and the literature review. Some indicators, driven by the theoretical models, are proposed in section 5. A measurement methodology is presented in section 6 and the conclusion, as well as the next steps, completes the document.

\section{Study background}

The main objective of this document is to propose a comprehensive review of the KPIs being proposed, as well as to provide the ideological model in terms of objectivity, then to take into account certain strong technological requirements and certain intelligent behaviors close to the human attitude for better interaction. In addition, it is proposed a procedure and a way to evaluate a component or sub-component of a smart city. The infrastructure put in place, as well as its ability to interact with its environment, will be essential elements to indicate the level of intelligence of a city.

In the case of developing countries, where a proper structure is still to be built, it is relevant to take into account additional requirements such as low and renewable energy consumption for the entire system, low cost of ownership, including edge computing, elasticity, availability, different communication options, 
etc. It would also be relevant to inculcate some properties into the systems to get closer to human behavior and to interact with people in a natural way.

\section{Research methodology}

The preparation of this article started with "What approach for Africa?" [14]. From the ITU (International Telecommunication Union) document [2] and [6], we were able to collect three more. Then, we built our search string as following: "Indicator" AND "Key Performance Indicator" AND "smartness" AND "smart city". IEEE Xplore, ScienceDirect and Google Scholar provided us respectively with 268, 95 and 44 results, from which we could extract only 04 deemed sufficient papers to carry out this work. In total, 9 relevant documents have made it possible to position this work with regards to the existing ones.

\section{Literature review}

Different approaches and proposals were encountered in the literature. The ITU [2] presents indicators as part of a holistic view of a city's performance in three dimensions; Economy, environment and society and culture. An example is the sub-dimension of the environment that provides a more detailed view of how nature is being used in a city. The Indicators it proposes measure progress on initiatives. For example, concerning the "Air Quality" category of the "environment" sub-component, its index is based on reported value for Particulate matter (PM10, and PM2.5), NO2 (nitrogen dioxide), SO2 (sulfur dioxide) and O3 (Ozone). The KPI (g / m3) is named "Air Pollution" and it takes as numerator, the mass of pollutant collected $(\mathrm{g})$ and as denominator, the volume of air sampled (m3). [3] Proposes a classification in a European context based on the challenges (impact on Urban Quality issues such as housing, economy, culture, social and environmental conditions that change the profile of a city and urban quality in its composition of factors and characteristics) related to competitiveness and sustainable urban development. It defines six (6) characteristics (smart economy, Smart People, smart Governance, smart Mobility, smart Environment, and Smart Living), each characteristic is estimated by a number of factors (33 in total) and each factor is described by a number of indicators (74 in total). [4] and [5] found that most of the proposed indicators are either of a political nature, or are not sustainable in the medium term, or focus on a particular area (technology, economy and environment, among others). To this end, they proposed the Cities in Motion Index (CIMI), which has been designed with the aim of constructing a "breakthrough" indicator in terms of completeness, characteristics, comparability and the quality and objectivity of its information. Its goal is to enable measurement of the future sustainability of the world's cities and the quality of life of their inhabitants. They conclude that CIMI aims to help the public and governments understand the performance of the 10 fundamental dimensions of a city: governance, urban planning, public management, technology, environment, international influence, social cohesion, mobility and 
transport, human capital and economy. It is also stated that all indicators are linked to a strategic objective that leads to a different type of local economic development: the creation of a global city, the promotion of entrepreneurship and innovation, among others.

Conversely, [7] states that no universally accepted index has been developed to assess intelligence in urban cities. It proposes the construction of an index called Smart City Index for Analytics (SMCIA), to objectively measure the degree of smartness in urban cities in six domain areas (smart living, smart people, smart mobility, smart economy, smart governance, smart environment) based on the Chinese context. As for [8] which, together with India's smart cities program, evaluated all the performance indicators set by International Telecommunications Union (ITU) to understand and analyze the number of needed indicators. For [9], emerging smart cities must be measured, monitored and managed in an optimal and intelligent way. It proposes the development of a classification framework of the performance indicators of a smart city based on the degree of objectivity of the observed variables and the level of technological advancement for data collection and applies it to the case of the municipality of Bari (Italy). Finally, [10] addressed the question of knowing to what extent can a set of smart city Key Performance Indicators according to the smart city literature be applied in practice?. The research question was then formulated to identify which indicators are used in the smart city literature and whether there is consensus or not. It is also discussed whether the measurement of these indicators is applicable and beneficial in practice. It then identified 62 theoretically judged indicators capable of measuring the performance of smart cities in the six areas of application of the smart city identified as smart governance, smart living, smart economy, smart environment, smart mobility, and smart people.

Overall, we surveyed key proposals for the performance measurement of the components of a smart city. It appears that there is a lack of consensus that can govern the paradigm. For the work, we will leverage the objective indicators that already exist and we will propose those that are necessary for the ideological framework. Then we will add the intrinsic indicators to take into account the technological factors typically, focusing on the requirements of the developing countries. These requirements will enable the indicators to acquire a better technological insight and will position them, step by step, in a sustainable and conventional way, towards smart and future cities. For that, it would also be very interesting to train the sub-components to learn from their data, then to offer useful services to the community.

\section{Indicators}

The objective indicators (or sub-components) $i_{j}$ (with $i$ who design the given component as defined in [15]), the technological factors $(T f)_{n}$ as well as the biological processes $(B P)_{m}$ will together constitute the KPIs and will make it possible to measure the smartness of the components. 


\subsection{Objective indicators}

A selective study was carried out and allowed for each given component, to propose a certain number of indicators that may be necessary to appreciate its objectivity [15](see Table 1).

Table 1. smart environment KPIs.

\begin{tabular}{|l|l|}
\hline $\mathrm{N}$ & KPI \\
\hline 1 & Air quality index (AQI) \\
2 & Greenhouse gas (GHG) emissions per capita \\
3 & Percentage of households covered by an audited Water Safety Plan \\
4 & Water consumption per capita \\
5 & Freshwater consumption \\
6 & Percentage of wastewater receiving treatment \\
7 & The percentage of solid waste dealt \\
8 & Percentage of mobile network antenna sites in compliance with EMF exposure guidelines \\
9 & Green area per 100,000 inhabitants \\
\hline
\end{tabular}

The objective indicators proposed for the ideological framework do not provide a complete view. Over time, it may be necessary to develop or supplement them. It will allow according to the framework, to position step by step towards the smart and future cities.

\subsection{Technological factors}

In addition to these objective indicators, we have defined a number of intrinsic indicators or technological factors enabling the developing country to position its cities towards smart and sustainable cities (see Table 2).

Table 2. Technological requirements.

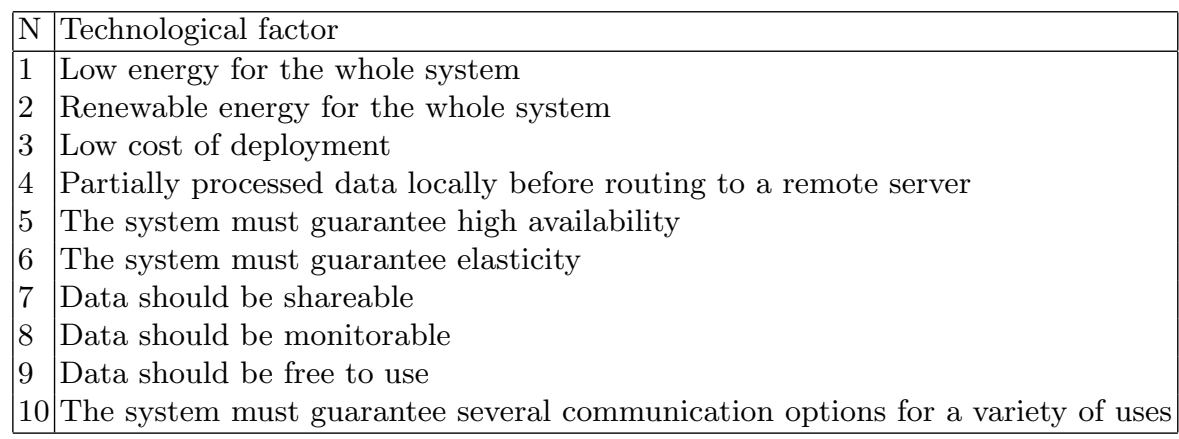




$$
T f=\sum_{n=1}^{10}(T f)_{n} \quad(T f)_{n}=0.1 \quad \text { or } \quad(T f)_{n}=0
$$

This is a threshold which takes the value 0.1 when the requirement is met, and 0 otherwise. The overall factor Tf (equation 1) is then the sum of the technological factors and is equal to 1 if all the technical requirements are taken into account.

\subsection{Biological processes}

It is also preferable to allow computer systems to perform one or more operations similar to learning and decision making by imitating biological processes, with particular emphasis on human cognitive processes [14]. At least one of the biological processes $(B P)_{m}$ such as learning, reasoning and self-correction can be taken into account (see Table 3 ).

Table 3. Biological processes.

\begin{tabular}{|l|l|}
\hline biological processes (BP) & Percentage by level of maturity \\
\hline learning & 0 to $100 \%$ \\
reasoning & 0 to $100 \%$ \\
self-correction & 0 to $100 \%$ \\
\hline
\end{tabular}

A system capable, after taking information on its operating model, to take preventive measures is undoubtedly the most ideal state. A percentage ranging from 0 (if it is not implemented) to 100 (according to its maturity) will be allocated to it as a good consideration.

The capacity of a system to learn from its data and then to reason in order to take into account all possible cases as well as a prediction (projection) in the near future, is fundamental. Learning and reasoning can each take a percentage between 0 and 100 according to their maturity also.

\section{Smartness measurement methodology}

Let $\mathrm{X}$ be given component, we denote by $X_{i}$ the $i$-th objective indicator of $\mathrm{X}$ and $P\left(X_{i}\right)$ its value in percentage. The smartness is measured in percentage. An intelligence level component $X$ may have several intelligence level subcomponents $X_{i}$. From intelligence level of a sub-component $I_{X_{i}}$, we can then calculate the intelligence level of a component $I_{X}$ by taking the summation of the $I_{X_{i}}$ and therefore the intelligence level of a city.

Technological factors are strictly objective and can provide the object ready to use. What will be more concrete is his gradual inculcation of certain cognitive processes. This is materialized by the equation 2 , for the sub-components. 


$$
I_{X_{i}}=\frac{T f * P\left(X_{i}\right)+\frac{1}{3}\left(\sum_{m=1}^{3}(B P)_{m}\right)}{2}
$$

\subsection{Illustration and simulation}

To illustrate, we will consider the "smart environment" component among the thirteen (13) defined in the ideological framework (see fig.1). This will help to position itself step by step towards the development of each component and therefore towards sustainable development for Smart and Future City. The evaluation of the other components will also follow the same principle.

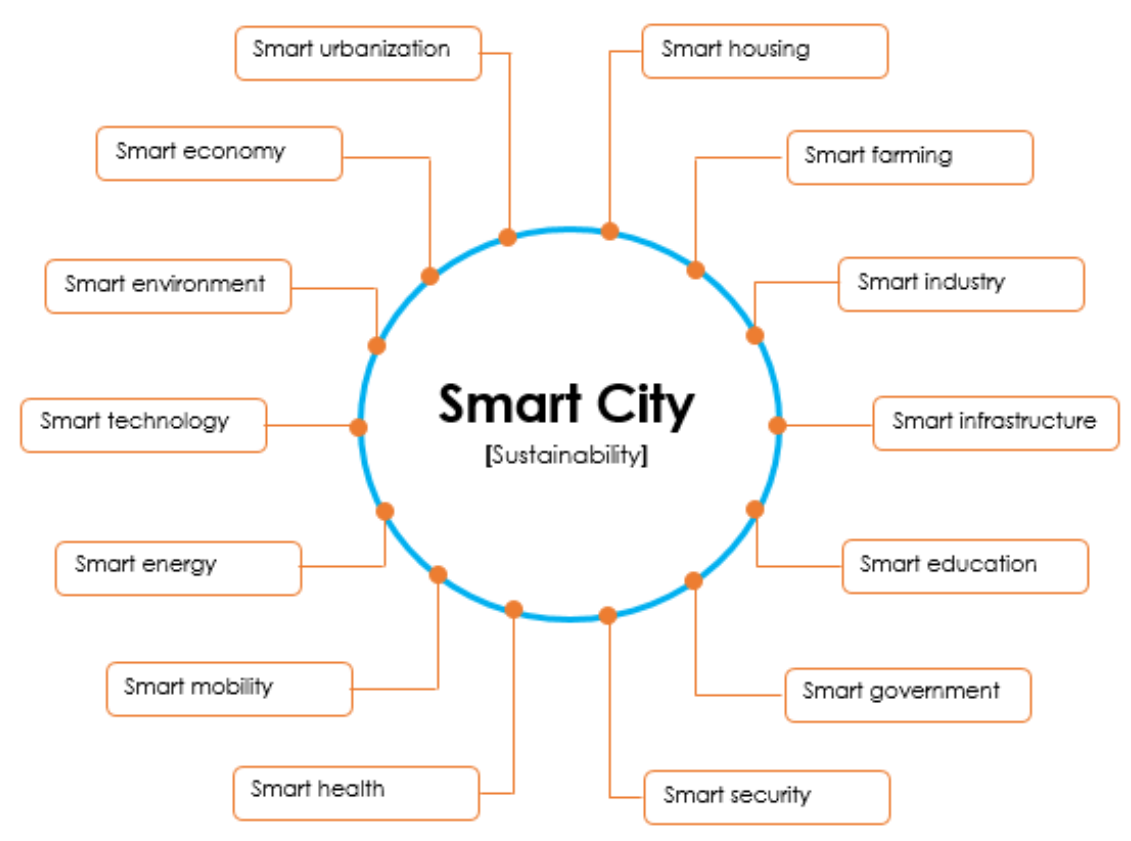

Fig. 1. the thirteen (13) defined in the ideological framework

It is a policy that, once adopted by a number of countries, will create competition and therefore be conducive to progress. This is how we considered using a city from each country in the ECOWAS region to better present the scenario. Noted that, Africa really remains another kind of context because, everything has to be almost built from scratch and so, deserves special attention. 
To be able to evaluate such a component, it is necessary that the city implements at least one sensor network with a minimum of technological requirements. The city must then implement intelligent tools for each sub-component and ensure the proper functioning of the entire system. As for the moment, this is not the case, we have, then, set up a simulator. Thus, we were able to simulate the intelligence of the component for the 15 countries.

Among the sub-components of the Smart Environment, the 'AQI' is not taken into account in the simulation because it is seen as a factor of quality. It tells us about the level of air pollution in a city. The sub-components 'Water consumption per capita' and 'Freshwater consumption' are not taken into account because they do not affect the result too much. We have, then, 6 objective indicators to consider:

- $\mathrm{P}\left(X_{1}\right)=(100$ - Percentage of greenhouse gas (GHG) emissions per capita)

$-\mathrm{P}\left(X_{2}\right)=$ Percentage of households covered by an audited Water Safety Plan

- $\mathrm{P}\left(X_{3}\right)=$ Percentage of wastewater receiving treatment

- $\mathrm{P}\left(X_{4}\right)=$ Percentage of solid waste dealt

$-\mathrm{P}\left(X_{5}\right)=$ Percentage of mobile network antenna sites in compliance with EMF exposure guidelines

- $\mathrm{P}\left(X_{6}\right)=$ Percentage of green area per 100,000 inhabitants

Table 4 and 5 give a suggested data generated for each city considered.

The results obtained [16] allow us to evaluate the level of progress, as regards the implementation of the "smart environment" component in accordance with this recommendation, by means of performance indicators. One can, then, affirm without doubt and according to the simulation (see fig.2), that the cities whose intelligence level is higher than average are those which are on a good level of development compared to those which are below.

These recommendations relate more to the digital policy of a management system of one or more countries wishing to put their cities in this direction. It can also be considered as a good decision-making tool, as a good tool for development assistance and as a good way to gradually move towards smart and future cities.

\section{Conclusion}

In short, intelligence embedded in an object deserves a lot of attention. The intelligence of a component begins to be felt when it grafts Information and Communication Technologies (ICT). This feeling refers to the impact of the system on its use. Objective indicators have been proposed, including by the ITU 
Table 4. Data generated for each city (part 1)

\begin{tabular}{|c|c|c|c|c|c|c|c|c|c|c|c|c|c|c|c|c|}
\hline city & $\mathrm{P}(X)$ & $T f_{1}$ & $T f_{2}$ & $T f_{3}$ & $T f_{4}$ & $T f_{5}$ & $T f_{6}$ & $T f_{7}$ & $T f_{8}$ & $T f_{9}$ & $T f_{10}$ & $B P_{1}$ & $B P_{2}$ & $B P_{3}$ & $I_{X_{i}}$ & $I_{X}$ \\
\hline \multirow{6}{*}{ DK } & $\mathrm{P}\left(X_{1}\right)=22$ & 0 & 0 & 0 & 0.1 & 0.1 & 0.1 & 0.1 & 0.1 & 0 & 0 & 56 & 17 & 60 & 27.67 & \multirow{6}{*}{$32.87 \%$} \\
\hline & $\mathrm{P}\left(X_{2}\right)=09$ & 0.1 & 0.1 & 0.1 & 0.1 & 0 & 0 & 0.1 & 0.1 & 0.1 & 0.1 & 29 & 77 & 99 & 37.76 & \\
\hline & $\mathrm{P}\left(X_{3}\right)=89$ & 0 & 0.1 & 0.1 & 0.1 & 0 & 0 & 0.1 & 0.1 & 0.1 & 0 & 39 & 64 & 72 & 55.87 & \\
\hline & $\mathrm{P}\left(X_{4}\right)=62$ & 0 & 0.1 & 0 & 0 & 0 & 0 & 0 & 0 & 0 & 0 & 66 & 46 & 48 & 29.77 & \\
\hline & $\mathrm{P}\left(X_{5}\right)=62$ & 0 & 0 & 0.1 & 0.1 & 0.1 & 0 & 0 & 0 & 0 & 0 & 25 & 37 & 24 & 23.63 & \\
\hline & $\mathrm{P}\left(X_{6}\right)=13$ & 0.1 & 0.1 & 0 & 0.1 & 0 & 0.1 & 0.1 & 0.1 & 0 & 0.1 & 34 & 59 & 15 & 22.55 & \\
\hline \multirow{6}{*}{ YM } & $\mathrm{P}\left(X_{1}\right)=46$ & 0.1 & 0.1 & 0 & 0 & 0 & 0 & 0.1 & 0.1 & 0 & 0.1 & 63 & 83 & 46 & 43.5 & \multirow{6}{*}{$40.55 \%$} \\
\hline & $\mathrm{P}\left(X_{2}\right)=12$ & 0 & 0 & 0 & 0 & 0 & 0.1 & 0.1 & 0 & 0.1 & 0 & 63 & 96 & 67 & 39.46 & \\
\hline & $\mathrm{P}\left(X_{3}\right)=24$ & 0 & 0.1 & 0 & 0.1 & 0.1 & 0 & 0.1 & 0.1 & 0.1 & 0.1 & 24 & 57 & 10 & 23.56 & \\
\hline & $\mathrm{P}\left(X_{4}\right)=88$ & 0.1 & 0.1 & 0.1 & 0.1 & 0.1 & 0.1 & 0.1 & 0.1 & 0 & 0 & 92 & 92 & 35 & 71.7 & \\
\hline & $\mathrm{P}\left(X_{5}\right)=41$ & 0 & 0.1 & 0 & 0 & 0.1 & 0 & 0.1 & 0.1 & 0 & 0.1 & 26 & 50 & 98 & 39.25 & \\
\hline & $\mathrm{P}\left(X_{6}\right)=01$ & 0.1 & 0 & 0 & 0.1 & 0.1 & 0.1 & 0 & 0.1 & 0.1 & 0 & 99 & 54 & 0 & 25.8 & \\
\hline \multirow{6}{*}{$\mathrm{CN}$} & $\mathrm{P}\left(X_{1}\right)=46$ & 0 & 0 & 0.1 & 0.1 & 0.1 & 0 & 0 & 0 & 0 & 0.1 & 99 & 45 & 40 & 39.87 & \multirow{6}{*}{$31.75 \%$} \\
\hline & $\mathrm{P}\left(X_{2}\right)=86$ & 0 & 0.1 & 0 & 0.1 & 0.1 & 0.1 & 0.1 & 0.1 & 0 & 0.1 & 63 & 53 & 33 & 54.93 & \\
\hline & $\mathrm{P}\left(X_{3}\right)=22$ & 0.1 & 0 & 0.1 & 0 & 0 & 0 & 0 & 0.1 & 0 & 0.1 & 12 & 23 & 80 & 23.57 & \\
\hline & $\mathrm{P}\left(X_{4}\right)=05$ & 0 & 0.1 & 0.1 & 0.1 & 0.1 & 0.1 & 0.1 & 0.1 & 0.1 & 0 & 4 & 70 & 77 & 27.17 & \\
\hline & $\mathrm{P}\left(X_{5}\right)=09$ & 0.1 & 0 & 0.1 & 0 & 0 & 0.1 & 0.1 & 0 & 0 & 0 & 8 & 4 & 80 & 17.13 & \\
\hline & $\mathrm{P}\left(X_{6}\right)=14$ & 0.1 & 0.1 & 0.1 & 0.1 & 0 & 0 & 0.1 & 0 & 0 & 0 & 57 & 86 & 3 & 27.83 & \\
\hline \multirow{6}{*}{ BS } & $\mathrm{P}\left(X_{1}\right)=38$ & 0.1 & 0 & 0.1 & 0.1 & 0.1 & 0 & 0.1 & 0.1 & 0.1 & 0.1 & 3 & 64 & 8 & 27.70 & \multirow{6}{*}{$34.29 \%$} \\
\hline & $\mathrm{P}\left(X_{2}\right)=16$ & 0.1 & 0.1 & 0 & 0 & 0.1 & 0.1 & 0.1 & 0.1 & 0.1 & 0.1 & 68 & 94 & 63 & 43.90 & \\
\hline & $\mathrm{P}\left(X_{3}\right)=25$ & 0 & 0.1 & 0.1 & 0.1 & 0 & 0.1 & 0 & 0 & 0.1 & 0.1 & 96 & 95 & 33 & 44.84 & \\
\hline & $\mathrm{P}\left(X_{4}\right)=45$ & 0.1 & 0 & 0 & 0 & 0.1 & 0 & 0 & 0 & 0 & 0.1 & 95 & 23 & 33 & 31.92 & \\
\hline & $\mathrm{P}\left(X_{5}\right)=11$ & 0.1 & 0.1 & 0 & 0 & 0 & 0 & 0 & 0.1 & 0.1 & 0 & 7 & 44 & 88 & 25.37 & \\
\hline & $\mathrm{P}\left(X_{6}\right)=87$ & 0 & 0 & 0 & 0 & 0 & 0 & 0 & 0.1 & 0 & 0 & 62 & 25 & 79 & 32.01 & \\
\hline \multirow{6}{*}{ BM } & $\mathrm{P}\left(X_{1}\right)=71$ & 0 & 0.1 & 0 & 0 & 0 & 0 & 0 & 0.1 & 0.1 & 0 & 89 & 56 & 43 & 41.98 & \multirow{6}{*}{$35.31 \%$} \\
\hline & $\mathrm{P}\left(X_{2}\right)=02$ & 0.1 & 0 & 0.1 & 0 & 0 & 0.1 & 0 & 0.1 & 0 & 0 & 40 & 67 & 63 & 28.73 & \\
\hline & $\mathrm{P}\left(X_{3}\right)=30$ & 0 & 0.1 & 0 & 0.1 & 0 & 0 & 0 & 0.1 & 0.1 & 0 & 58 & 45 & 2 & 23.50 & \\
\hline & $\mathrm{P}\left(X_{4}\right)=57$ & 0.1 & 0.1 & 0.1 & 0 & 0.1 & 0.1 & 0.1 & 0 & 0.1 & 0 & 78 & 7 & 22 & 37.78 & \\
\hline & $\mathrm{P}\left(X_{5}\right)=22$ & 0.1 & 0.1 & 0.1 & 0.1 & 0 & 0 & 0.1 & 0.1 & 0 & 0.1 & 9 & 83 & 34 & 28.70 & \\
\hline & $\mathrm{P}\left(X_{6}\right)=56$ & 0 & 0 & 0.1 & 0 & 0.1 & 0.1 & 0 & 0 & 0.1 & 0.1 & 63 & 93 & 67 & 51.16 & \\
\hline \multirow{6}{*}{ CT } & $\mathrm{P}\left(X_{1}\right)=08$ & 0.1 & 0 & 0 & 0.1 & 0 & 0 & 0.1 & 0 & 0 & 0 & 6 & 78 & 33 & 20.7 & \multirow{6}{*}{$38.44 \%$} \\
\hline & $\mathrm{P}\left(X_{2}\right)=61$ & 0.1 & 0.1 & 0 & 0 & 0.1 & 0 & 0.1 & 0.1 & 0.1 & 0.1 & 21 & 92 & 1 & 40.35 & \\
\hline & $\mathrm{P}\left(X_{3}\right)=28$ & 0 & 0.1 & 0 & 0 & 0 & 0 & 0 & 0.1 & 0 & 0.1 & 37 & 98 & 2 & 27.03 & \\
\hline & $\mathrm{P}\left(X_{4}\right)=14$ & 0 & 0.1 & 0 & 0 & 0 & 0.1 & 0 & 0.1 & 0 & 0.1 & 93 & 71 & 16 & 32.80 & \\
\hline & $\mathrm{P}\left(X_{5}\right)=91$ & 0.1 & 0.1 & 0.1 & 0 & 0 & 0.1 & 0.1 & 0 & 0.1 & 0 & 58 & 30 & 17 & 44.80 & \\
\hline & $\mathrm{P}\left(X_{6}\right)=85$ & 0.1 & 0.1 & 0 & 0.1 & 0.1 & 0 & 0.1 & 0.1 & 0.1 & 0.1 & 42 & 68 & 76 & 65.00 & \\
\hline \multirow{6}{*}{ LM } & $\mathrm{P}\left(X_{1}\right)=30$ & 0.1 & 0 & 0 & 0 & 0 & 0.1 & 0.1 & 0.1 & 0.1 & 0.1 & 90 & 39 & 69 & 42.00 & \multirow{6}{*}{$34.50 \%$} \\
\hline & $\mathrm{P}\left(X_{2}\right)=34$ & 0 & 0 & 0 & 0 & 0 & 0.1 & 0 & 0.1 & 0 & 0 & 47 & 68 & 11 & 24.40 & \\
\hline & $\mathrm{P}\left(X_{3}\right)=51$ & 0.1 & 0 & 0.1 & 0.1 & 0.1 & 0.1 & 0.1 & 0.1 & 0.1 & 0.1 & 89 & 42 & 51 & 53.28 & \\
\hline & $\mathrm{P}\left(X_{4}\right)=50$ & 0.1 & 0 & 0.1 & 0.1 & 0.1 & 0 & 0.1 & 0 & 0 & 0.1 & 89 & 41 & 9 & 38.17 & \\
\hline & $\mathrm{P}\left(X_{5}\right)=55$ & 0 & 0.1 & 0.1 & 0.1 & 0.1 & 0 & 0.1 & 0.1 & 0 & 0 & 65 & 4 & 50 & 36.33 & \\
\hline & $\mathrm{P}\left(X_{6}\right)=01$ & 0.1 & 0.1 & 0 & 0 & 0 & 0 & 0 & 0 & 0 & 0.1 & 8 & 42 & 26 & 12.81 & \\
\hline \multirow{6}{*}{$\mathrm{CC}$} & $\mathrm{P}\left(X_{1}\right)=67$ & 0 & $\mid 0.1$ & 0 & 0 & 0 & 0.1 & 0 & 0 & 0.1 & 0 & 72 & 23 & 37 & 32.05 & \multirow{6}{*}{$41.31 \%$} \\
\hline & $\mathrm{P}\left(X_{2}\right)=73$ & 0 & 0.1 & 0.1 & 0.1 & 0.1 & 0.1 & 0 & 0.1 & 0 & 0.1 & 72 & 88 & 1 & 52.38 & \\
\hline & $\mathrm{P}\left(X_{3}\right)=53$ & 0 & 0.1 & 0.1 & 0 & 0.1 & 0.1 & 0.1 & 0.1 & 0 & 0.1 & 31 & 54 & 99 & 46.56 & \\
\hline & $\mathrm{P}\left(X_{4}=99\right.$ & 0 & 0 & 0.1 & 0.1 & 0.1 & 0 & 0 & 0 & 0 & 0.1 & 23 & 30 & 50 & 36.97 & \\
\hline & $\mathrm{P}\left(X_{5}\right)=85$ & 0 & 0 & 0.1 & 0.1 & 0.1 & 0 & 0.1 & 0.1 & 0 & 0 & 11 & 93 & 43 & 45.75 & \\
\hline & $\mathrm{P}\left(X_{6}\right)=83$ & 0 & 0 & 0 & 0.1 & 0 & 0 & 0 & 0.1 & 0 & 0.1 & 58 & 38 & 34 & 34.12 & \\
\hline
\end{tabular}


Table 5. Data generated for each city (part 2)

\begin{tabular}{|c|c|c|c|c|c|c|c|c|c|c|c|c|c|c|c|c|}
\hline city & $\mathrm{P}(X)$ & $T f_{1}$ & $T f_{2}$ & $T f_{3}$ & $T f_{4}$ & $T f_{5}$ & $T f_{6}$ & $T f_{7}$ & $T f_{8}$ & $T f_{9}$ & $T f_{10}$ & $B P_{1}$ & $B P_{2}$ & $B P_{3}$ & $I_{X_{i}}$ & $I_{X}$ \\
\hline \multirow{6}{*}{ BJ } & $\mathrm{P}\left(X_{1}\right)=56$ & 0.1 & 0 & 0 & 0 & 0 & 0.1 & 0.1 & 0 & 0.1 & 0 & 29 & 54 & 52 & 33.70 & \multirow{6}{*}{$27.80 \%$} \\
\hline & $\mathrm{P}\left(X_{2}\right)=34$ & 0.1 & 0 & 0 & 0 & 0.1 & 0 & 0 & 0.1 & 0.1 & 0.1 & 11 & 22 & 93 & 29.50 & \\
\hline & $\mathrm{P}\left(X_{3}\right)=10$ & 0 & 0 & 0.1 & 0.1 & 0 & 0.1 & 0 & 0 & 0.1 & 0.1 & 98 & 18 & 17 & 24.67 & \\
\hline & $\mathrm{P}\left(X_{4}\right)=06$ & 0 & 0 & 0 & 0.1 & 0 & 0.1 & 0.1 & 0.1 & 0 & 0.1 & 7 & 43 & 53 & 18.67 & \\
\hline & $\mathrm{P}\left(X_{5}\right)=03$ & 0.1 & 0.1 & 0 & 0 & 0 & 0.1 & 0 & 0 & 0.1 & 0 & 44 & 40 & 17 & 17.43 & \\
\hline & $\mathrm{P}\left(X_{6}\right)=79$ & 0 & 0.1 & 0 & 0 & 0 & 0.1 & 0 & 0.1 & 0 & 0 & 79 & 32 & 75 & 42.85 & \\
\hline \multirow{6}{*}{$\mathrm{LG}$} & $\mathrm{P}\left(X_{1}\right)=03$ & 0 & 0 & 0.1 & 0.1 & 0 & 0 & 0 & 0 & 0.1 & 0.1 & 57 & 47 & 25 & 22.10 & \multirow{6}{*}{$29.73 \%$} \\
\hline & $\mathrm{P}\left(X_{2}\right)=57$ & 0.1 & 0 & 0.1 & 0.1 & 0.1 & 0.1 & 0 & 0 & 0.1 & 0 & 0 & 27 & 5 & 22.43 & \\
\hline & $\mathrm{P}\left(X_{3}\right)=19$ & 0.1 & 0 & 0.1 & 0 & 0.1 & 0.1 & 0.1 & 0 & 0.1 & 0 & 18 & 29 & 43 & 20.70 & \\
\hline & $\mathrm{P}\left(X_{4}\right)=08$ & 0.1 & 0.1 & 0.1 & 0 & 0 & 0 & 0 & 0.1 & 0.1 & 0.1 & 87 & 54 & 39 & 32.40 & \\
\hline & $\mathrm{P}\left(X_{5}\right)=56$ & 0.1 & 0 & 0.1 & 0 & 0.1 & 0 & 0.1 & 0 & 0.1 & 0.1 & 40 & 27 & 93 & 43.47 & \\
\hline & $\mathrm{P}\left(X_{6}\right)=08$ & 0 & 0.1 & 0 & 0 & 0 & 0.1 & 0.1 & 0 & 0.1 & 0 & 95 & 32 & 87 & 37.26 & \\
\hline \multirow{6}{*}{$\mathrm{PR}$} & $\mathrm{P}\left(X_{1}\right)=59$ & 0.1 & 0 & \begin{tabular}{|l|}
0.1 \\
\end{tabular} & 0 & 0 & 0.1 & 0.1 & \begin{tabular}{|l|}
0.1 \\
\end{tabular} & 0.1 & 0.1 & 59 & 92 & 22 & 49.48 & \multirow{6}{*}{$31.96 \%$} \\
\hline & $\mathrm{P}\left(X_{2}\right)=94$ & 0.1 & 0.1 & 0.1 & 0 & 0 & 0.1 & 0 & 0 & 0 & 0.1 & 51 & 26 & 3 & 36.83 & \\
\hline & $\mathrm{P}\left(X_{3}\right)=11$ & 0 & 0.1 & 0 & 0 & 0.1 & 0.1 & 0.1 & 0 & 0 & 0.1 & 73 & 80 & 23 & 32.08 & \\
\hline & $\mathrm{P}\left(X_{4}\right)=06$ & 0.1 & 0.1 & 0.1 & 0.1 & 0.1 & 0 & 0 & 0.1 & 0.1 & 0 & 3 & 14 & 50 & 13.26 & \\
\hline & $\mathrm{P}\left(X_{5}\right)=11$ & 0.1 & 0.1 & 0 & 0 & 0 & 0.1 & 0 & 0.1 & 0 & 0 & 35 & 25 & 19 & 15.37 & \\
\hline & $\mathrm{P}\left(X_{6}\right)=91$ & 0 & 0 & 0 & 0 & 0.1 & 0 & 0.1 & 0.1 & 0.1 & 0 & 55 & 48 & 56 & 44.70 & \\
\hline \multirow{6}{*}{ NM } & $\mathrm{P}\left(X_{1}\right)=11$ & 0.1 & 0.1 & 0 & 0.1 & 0.1 & 0.1 & 0.1 & 0 & 0.1 & 0 & 80 & 50 & 93 & 41.01 & \multirow{6}{*}{$40.70 \%$} \\
\hline & $\mathrm{P}\left(X_{2}\right)=45$ & 0 & 0.1 & 0 & 0.1 & 0 & 0 & 0 & 0 & 0.1 & 0.1 & 61 & 83 & 23 & 36.83 & \\
\hline & $\mathrm{P}\left(X_{3}\right)=97$ & 0.1 & 0.1 & 0.1 & 0.1 & 0.1 & 0 & 0.1 & 0 & 0.1 & 0.1 & 6 & 55 & 7 & 50.13 & \\
\hline & $\mathrm{P}\left(X_{4}\right)=29$ & 0.1 & 0.1 & 0.1 & 0 & 0 & 0.1 & 0 & 0 & 0 & 0 & 22 & 81 & 53 & 31.80 & \\
\hline & $\mathrm{P}\left(X_{5}\right)=58$ & 0 & 0.1 & 0 & 0 & 0 & 0.1 & 0 & 0 & 0.1 & 0.1 & 67 & 48 & 1 & 30.93 & \\
\hline & $\mathrm{P}\left(X_{6}\right)=68$ & 0 & 0 & 0 & 0.1 & 0 & 0 & 0.1 & 0.1 & 0.1 & 0 & 58 & 90 & 91 & 53.43 & \\
\hline \multirow{6}{*}{ MR } & $\mathrm{P}\left(X_{1}\right)=63$ & 0 & 0 & 0 & 0 & 0.1 & 0 & 0.1 & \begin{tabular}{|l|}
0.1 \\
\end{tabular} & 0 & 0 & 34 & 67 & 33 & 31.78 & \multirow{6}{*}{$36.98 \%$} \\
\hline & $\mathrm{P}\left(X_{2}\right)=11$ & 0.1 & 0 & 0 & 0.1 & 0.1 & 0.1 & 0.1 & 0.1 & 0 & 0.1 & 91 & 85 & 96 & 49.18 & \\
\hline & $\mathrm{P}\left(X_{3}\right)=52$ & 0.1 & 0.1 & 0 & 0.1 & 0.1 & 0 & 0.1 & 0.1 & 0 & 0.1 & 37 & 59 & 28 & 38.87 & \\
\hline & $\mathrm{P}\left(X_{4}\right)=34$ & 0.1 & 0 & 0 & 0.1 & 0 & 0 & 0.1 & 0.1 & 0.1 & 0.1 & 52 & 45 & 25 & 30.53 & \\
\hline & $\mathrm{P}\left(X_{5}\right)=11$ & 0.1 & 0 & 0.1 & 0 & 0.1 & 0.1 & 0 & 0 & 0 & 0.1 & 11 & 87 & 39 & 25.58 & \\
\hline & $\mathrm{P}\left(X_{6}\right)=57$ & 0 & 0.1 & 0 & 0.1 & 0 & 0.1 & 0 & 0 & 0.1 & 0.1 & 82 & 32 & 76 & 45.92 & \\
\hline \multirow{6}{*}{$\mathrm{FT}$} & $\mathrm{P}\left(X_{1}\right)=68$ & 0 & 0.1 & \begin{tabular}{|l|l|}
0.1 & \\
\end{tabular} & 0.1 & 0 & 0 & 0.1 & 0.1 & 0.1 & 0 & 10 & 13 & 54 & 33.23 & \multirow{6}{*}{$40.97 \%$} \\
\hline & $\mathrm{P}\left(X_{2}\right)=86$ & 0.1 & 0.1 & 0 & 0.1 & 0.1 & 0 & 0 & 0 & 0.1 & 0.1 & 84 & 88 & 95 & 70.30 & \\
\hline & $\mathrm{P}\left(X_{3}\right)=92$ & 0 & 0 & 0.1 & 0 & 0 & 0 & 0 & 0.1 & 0 & 0 & 87 & 78 & 33 & 42.20 & \\
\hline & $\mathrm{P}\left(X_{4}\right)=10$ & 0 & 0.1 & 0.1 & 0.1 & 0.1 & 0.1 & 0.1 & 0 & 0 & 0 & 21 & 91 & 33 & 27.16 & \\
\hline & $\mathrm{P}\left(X_{5}\right)=51$ & 0 & 0 & 0.1 & 0.1 & 0 & 0.1 & 0.1 & 0.1 & 0.1 & 0.1 & 17 & 93 & 0 & 36.18 & \\
\hline & $\mathrm{P}\left(X_{6}\right)=18$ & 0.1 & 0.1 & 0.1 & 0 & 0 & 0.1 & 0.1 & 0.1 & 0 & 0 & 79 & 24 & 85 & 36.73 & \\
\hline \multirow{6}{*}{ GD } & $\mathrm{P}\left(X_{1}\right)=31$ & 0 & 0.1 & 0.1 & 0 & 0 & 0.1 & 0 & 0 & 0 & 0.1 & 26 & 91 & 52 & 34.37 & \multirow{6}{*}{$34.05 \%$} \\
\hline & $\mathrm{P}\left(X_{2}\right)=89$ & 0 & 0 & 0.1 & 0 & 0.1 & 0 & 0 & 0.1 & 0 & 0.1 & 78 & 2 & 82 & 44.80 & \\
\hline & $\mathrm{P}\left(X_{3}\right)=29$ & 0 & 0 & 0 & 0.1 & 0 & 0.1 & 0 & 0 & 0 & 0 & 47 & 44 & 48 & 26.07 & \\
\hline & $\mathrm{P}\left(X_{4}\right)=40$ & 0.1 & 0.1 & 0.1 & 0 & 0.1 & 0 & 0.1 & 0 & 0 & 0 & 90 & 42 & 31 & 37.17 & \\
\hline & $\mathrm{P}\left(X_{5}\right)=23$ & 0 & 0 & 0.1 & 0 & 0.1 & 0.1 & 0.1 & 0 & 0 & 0.1 & 58 & 57 & 82 & 38.58 & \\
\hline & $\mathrm{P}\left(X_{6}\right)=24$ & 0.1 & 0 & 0 & 0 & 0.1 & 0.1 & 0 & 0 & 0.1 & 0.1 & 28 & 58 & 18 & 23.33 & \\
\hline
\end{tabular}

Table4: Dakar (DK), Yamoussoukro (YM), Conakry (CN), Bissau (BS), Bamako (BM), Cotonou (CT), Lome (LM), Accra (CC).

Table5: Banjul (BJ), LagosS (LG), Praia (PR), Niamey (NM), Monrovia (MR), Freetown (FT), Ouagadougou (GD). 

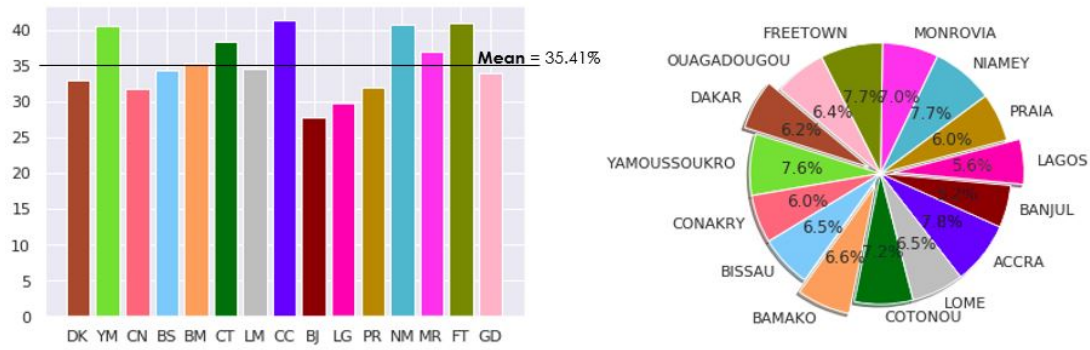

Fig. 2. Progress of the cities concerning the implementation of the "smart environment" component

and others, and will enable cities to measure their progress over time, compare their performance with that of other cities and achieve the Sustainable Development Goals. In addition to the objective indicators, we have identified a number of technological requirements, known as technology factors that can impact sustainability and play a consensual role in implementing a smart city component. Some cognitive processes have also been taken into account in order to give the term intelligence its true meaning in this paradigm.

This is the logical continuation of the first step of our work on "What approach for Africa?" [14] and proposes a way to evaluate a smart city component defined in the "ideological framework" taking into account technological requirements in the context of developing countries. Especially those who will have everything to build, as is the case for most African countries. This will propel them towards sustainability and will also enable them to position themselves on a consensual evolution towards smart and future cities.

This study will enable us, in future to evaluate our future work on well-defined aspects of a given component. Since infrastructure is almost non-existent, the next step will be topropose and implement architectures that are affordable, accessibleand taking into account the reality of developing countries.

\section{References}

1. Mohanty, S. P., Choppali, U., Kougianos, E.: Everything you wanted to know about smart cities: The Internet of things is the backbone. IEEE Consumer Electronics Magazine, 60-70 (2016). https://doi.org/10.1109/MCE.2016.2556879

2. Collection Methodology for Key Performance Indicators for Smart, https://www.itu.int/en/publications/Documents/tsb/2017-U4SSC-CollectionMethodology/index.html. Last accessed 15 March 2019 
3. Smart Cities: Ranking of European Medium-Sized Cities, http://www.smartcities.eu/download/smart_cities_final_report.pdf. Last accessed 15 March 2019

4. IESE Cities in Motion. Cities in motion Index 2014, http://www.iese.edu/research/pdfs/ST-0396-E.pdf. Last accessed 15 March 2019

5. IESE Cities in Motion. Cities in motion Index 2015, http://www.iese.edu/research/pdfs/ST-0366-E.pdf. Last accessed 15 March 2019

6. Mila Gasc-Hernandez: Building a smart city: lessons from Barcelona. Magazine Communications of the ACM, 50-57 (2018). https://doi.org/10.1145/3117800

7. Yin-Leng Theng, Xuexin $\mathrm{Xu}$, Witedwittayanusat Kanokkorn: Towards the Construction of Smart City Index for Analytics (SM-CIA): Pilot-Testing with Major Cities in China Using Publicly Available Data. In: 49th Hawaii International Conference on System Sciences (HICSS), pp. 2964-2973, IEEE (2016). https://doi.org/10.1109/HICSS.2016.371

8. Srinjoy Nag Chowdhury, Saniya Dhawan: Evaluation of key performance indicators of smartcities by Delphi analysis. In: IEEE International Conference on Recent Trends in Electronics, Information Communication Technology (RTEICT), pp. 337342, IEEE (2016). https://doi.org/10.1109/RTEICT.2016.7807838

9. Raffaele Carli, Mariagrazia Dotoli, Roberta Pellegrino, Luigi Ranieri: Measuring and Managing the Smartness of Cities: A Framework for Classifying Performance Indicators. In: IEEE International Conference on Systems, Man, and Cybernetics, pp. 1288 - 1293, IEEE (2013). https://doi.org/10.1109/SMC.2013.223

10. Unfolding the Smart City Label: Definition and Application of Performance Measurement System for Smart Cities, https://pdfs.semanticscholar.org/0e08/5870dd884114ccbf846e6354fbdeef61a3ed.pdf. Last accessed 15 March 2019

11. Indicators of sustainable agriculture: a scoping analysis, https://www.wri.org/sites/default/files/wrr_installment_6_sustainable_agruiculture_ indicators.pdf. Last accessed 15 March 2019

12. The Singapore Smart Industry Readiness Index: Catalysing the transformation of manufacturing, https://www.gov.sg/ /sgpcmedia/media_releases/edb/press_release/P-201711131/attachment/The\%20Singapore\%20Smart\%20Industry\%20Readiness \%20Index\%20\%20Whitepaper_final.pdf. Last accessed 15 March 2019

13. 25 indicateurs de performance industrielle pour les units de production, https://blog.fr-techteam.com/25-indicateurs-de-performance-industrielle-pour-lesunites-de-production. Last accessed 15 March 2019

14. BARRO P. A., DEGILA J., ZENNARO M., WAMBA, S. F.: Towards Smart and Sustainable Future Cities Based on Internet of Things for Developing Countries: What Approach for Africa?. EAI (2018). https://doi.org/10.4108/eai.11-92018.155481

15. BASICS KPI FOR SMART CITY COMPONENTS (SMART X), https://github.com/pape-barro/basics_kpi/wiki/BASICS-KPI-FOR-SMARTCITY-COMPONENTS-(SMART-X). Last accessed 15 March 2019

16. simulation and evaluation of the 'smart environment' component, https://github.com/papebarro/basics ${ }_{k}$ pi/blob/master/kpis.ipynb.Lastaccessed15March2019 